\title{
Intraarticular Osteoid Osteoma: MRI Characteristics and Clinical Presentation Before and After Radiofrequency Ablation Compared to Extraarticular Osteoid Osteoma
}

\section{Das intraartikuläre Osteoidosteom: MRT-Charakteristik und klinisches Erscheinungsbild vor und nach Radiofrequenzablation im Vergleich zum extraartikulären Osteoidosteom}

Authors

Thomas Germann ${ }^{1}$, Marc-André Weber ${ }^{2}$, Burkhard Lehner ${ }^{3}$, Laurent Kintzele ${ }^{1}$, Iris Burkholder ${ }^{4}$, Hans-Ulrich Kauczor ${ }^{1}$, Christoph Rehnitz ${ }^{1}$

Affiliations

1 Diagnostic and Interventional Radiology, University Hospital Heidelberg, Germany

2 Diagnostic and Interventional Radiology, University Hospital Rostock, Germany

3 Department of Orthopaedics Emergency Surgery and Paraplegia, University Hospital Heidelberg, Germany

4 StaBiL, Statistical and Biometric Solutions, Zweibrücken, Germany

Key words osteoid osteoma, intraarticular, radiofrequency (RF) ablation, MRI

received 03.10.2019

accepted 11.04.2020

published online 08.07 .2020

Bibliography

Fortschr Röntgenstr 2020; 192: 1190-1198

DOI 10.1055/a-1181-9041

ISSN 1438-9029

(C) 2020. Thieme. All rights reserved.

Georg Thieme Verlag KG, Rüdigerstraße 14,

70469 Stuttgart, Germany

Correspondence

Dr. Thomas Germann

Diagnostische und Interventionelle Radiologie,

Universitätsklinikum Heidelberg, Im Neuenheimer Feld 110, 69120 Heidelberg, Germany

Tel.: ++49/62 21/5634680

Thomas.Germann@med.uni-heidelberg.de

\section{ABSTRACT}

Purpose To determine MRI characteristics and the clinical presentation of intraarticular osteoid osteomas (OO) before and after treatment with $\mathrm{CT}$-guided radiofrequency ablation (RFA) compared with extraarticular osteoid osteomas.
Materials and Methods In a retrospective study, $\mathrm{n}=21$ patients with an intraarticular $\mathrm{OO}$ were matched with a control group of $\mathrm{n}=21$ patients with an extraarticular $\mathrm{OO}$ at a comparable anatomical position. All patients underwent CT-guided RFA and preinterventional MRI. In $n=31$ cases, follow-up MR imaging was available. MR images were analyzed for morphologic features: effusion and synovitis, bone marrow edema (BME), soft tissue edema, periosteal reaction as well as T1 / T2 signal and contrast enhancement of the nidus. Recorded clinical parameters included the initial diagnosis, the course of pain symptoms after RFA and the incidence of complications.

Results The nidus was detectable in all patients on MRI. BME had the highest sensitivity in both intra- and extraarticular $\mathrm{OO}$ (100\%). Effusion and synovitis were only observed in the intraarticular $\mathrm{OO}$ group $(\mathrm{n}=21)$ with a perfect sensitivity and specificity $(100 \%)$ and a high negative predictive value ( $85 \%)$. Soft tissue edema was significantly more present in patients with intraarticular $O O(p=0.0143)$. No significant differences were present regarding periosteal reaction, $\mathrm{T} 1 / \mathrm{T} 2$ signal and contrast enhancement of the nidus ( $p>0.05$ ). BME, contrast enhancement, soft tissue edema, periosteal reaction, effusion and synovitis, if preexisting, always decreased after RFA. In $66.7 \%$ of patients with intraarticular $\mathrm{OO}$, a false initial diagnosis was made (extraarticular: $19 \%$ ). All patients were free of pain after intervention. Complications following the RFA procedure did not occur.

Conclusion MRI demonstrates the nidus and thus the $\mathrm{OO}$ in all cases regardless of the location. The characteristic MRI morphology of an intraarticular $\mathrm{OO}$ includes synovitis and joint effusion, which are always present and differentiate with perfect sensitivity/specificity from an extraarticular 00 . In both intra- and extraarticular OOs pathologic MRI changes at least decreased or completely normalized and the clinical results after RFA were excellent.

Key Points:

- MRI is excellently suited for the diagnosis of intra- and extraarticular OOs.

- Joint effusion and synovitis distinguish both forms with perfect sensitivity and specificity. 
- All MRI changes, which indicate activity, decreased after successful RFA.

- The clinical results after RFA are excellent in both forms.

\section{Citation Format}

- Germann T, Weber M, Lehner B et al. Intraarticular Osteoid Osteoma: MRI Characteristics and Clinical Presentation Before and After Radiofrequency Ablation Compared to Extraarticular Osteoid Osteoma. Fortschr Röntgenstr 2020; 192: 1190-1198

\section{ZUSAMMENFASSUNG}

Ziel Untersucht wurden MRT-Charakteristik und klinisches Erscheinungsbild von intraartikulären Osteoidosteomen (OO) vor und nach der Behandlung mit Radiofrequenzablation (RFA) im Vergleich zu extraartikulären OO.

Material und Methoden In einer retrospektiven Studie wurden $n=21$ Patienten mit intraartikulärem 00 einer gleichgroßen Kontrollgruppe von $n=21$ Patienten mit extraartikulärem $\mathrm{OO}$ an vergleichbarer anatomischer Position gegenübergestellt. Alle Patienten erhielten eine CT-gesteuerte RFA sowie eine präinterventionelle MRT. Bei $n=31$ Patienten lag eine Verlaufsbildgebung vor. Die MRT-Bilder wurden hinsichtlich morphologischer Merkmale analysiert: Erguss und Synovitis, Knochenödem, Weichteilödem, Periostreaktion sowie T1-/T2-Signal und Kontrastmittel (KM)-Aufnahme des Nidus. Erfasste klinische Parameter waren unter anderem die initiale Verdachtsdiagnose, der Verlauf der Schmerzsymptomatik nach RFA und das Auftreten von Komplikationen.
Ergebnisse Der Nidus war bei allen Patienten in der MRT erkennbar. Das Knochenödem wies die höchste Sensitivität sowohl beim intra- als auch beim extraartikulären 00 auf (100\%). Erguss und Synovitis wurden nur in der Gruppe der intraartikulären $\mathrm{OO}(\mathrm{n}=21)$ beobachtet mit einer perfekten Sensitivität und Spezifität (100\%) und einem hohen negativen Vorhersagewert (85\%). Bei Patienten mit intraartikulärem OO war ein Weichteilödem signifikant häufiger vorhanden $(p=0,0143)$. Periostreaktion, T1-/T2-Signal und KM-Aufnahme des Nidus zeigten keine signifikanten Unterschiede $(p>0,05)$. Knochenödem, KM-Aufnahme, Weichteilödem, Periostreaktion, Erguss und Synovitis waren, sofern vorbestehend, immer rückläufig nach RFA. Bei 66,7\% der Patienten mit intraartikulärem $\mathrm{OO}$ wurde initial eine falsche Verdachtsdiagnose gestellt (extraartikulär: 19\%). Alle Patienten waren nach dem Eingriff schmerzfrei. Komplikationen traten nicht auf.

Schlussfolgerung Die MRT zeigt den Nidus und damit das $\mathrm{OO}$ in allen Fällen unabhängig von der Lokalisation. Die charakteristische MRT-Morphologie des intraartikulären $\mathrm{OO}$ umfasst die Synovitis und den Gelenkerguss, die immer vorliegen und mit perfekter Sensitivität/Spezifität von einem extraartikulären $\mathrm{OO}$ differenzieren. Sowohl beim intra- als auch beim extraartikulären $\mathrm{OO}$ waren die pathologischen MRT-Veränderungen mindestens abnehmend oder vollständig normalisiert und die klinischen Ergebnisse nach RFA waren ausgezeichnet.

\section{Introduction}

Osteoid osteoma (OO) is a common bone tumor and accounts for approximately $10 \%$ of all benign bone lesions as well as $3 \%$ of all primary bone tumors [1]. It usually occurs in the second or third decade of life. Men are affected slightly more frequently than women (3:1) [2]. It is primarily located in the metaphyses and diaphyses of the long bones. However, approximately $13 \%$ of OOs are intraarticular [2]. While there are numerous studies on extraarticular OOs with large case numbers, intraarticular OOs have primarily only been included in case reports, with the most common manifestations being in the hip joint $[3,4]$, the elbow joint [5] and the ankle joint [6]. The clinical and morphological appearance of an intraarticular $\mathrm{OO}$ is often atypical and differs from that of an extraarticular $\mathrm{OO}$ [7]. The published case reports usually showed an incorrect initial primary diagnosis. Nonspecific symptoms like limited mobility, flexion deformities, muscle atrophy, and arthritic changes are not uncommon [8]. The typical sclerosis is less pronounced on CT $[9,10]$ and scintigraphy may not show the classic features [11] so that the nidus cannot always be definitively identified. This presents a diagnostic challenge and can greatly delay correct diagnosis as well as any necessary treatment. However, early intervention is extremely important particularly in the case of intraarticular $\mathrm{OO}$ to avoid potential subsequent damage like skeletal deformities and irreversible defects of the articular cartilage due to chronic synovitis [12]. Computed tomography (CT)-guided radiofrequency ablation (RFA) is an efficient treatment option [13]. Magnetic resonance imaging (MRI) has become a valuable tool for the diagnosis of $\mathrm{OO}$ since it can easily visualize the nidus and reactions in the surrounding areas such as bone marrow edema and soft tissue edema as well as joint effusion and synovitis [14]. This study examines the MRI features and clinical characteristics of intraarticular compared to extraarticular $\mathrm{OO}$ and their occurrence before and after RFA.

\section{Materials and Methods}

\section{Ethical principles, inclusion, and exclusion criteria}

This study was approved by the ethics committee. After a thorough patient briefing regarding the type of procedure and possible risks, all patients provided written informed consent prior to the examination. The present study was performed in its current form in accordance with the Declaration of Helsinki. All patients with intraarticular OO who underwent CT-guided RFA in our institution between January 2009 and December 2018 and who had undergone a prior MRI examination were included in the study. The same number of consecutive patients with extraarticular $\mathrm{OO}$ in a comparable anatomical location with an available 
preinterventional MRI examination were included as a comparison group. In addition, a preinterventional CT image dataset was available for all patients. The diagnosis of $\mathrm{OO}$ was made based on the clinical presentation and morphological criteria. The most important diagnostic criterion was the detection of a nidus which was successful in all cases on MRI and was able to be confirmed on $\mathrm{CT}$. In agreement with other work groups, histological confirmation was not used for diagnosis $[15,16]$. Patients with other underlying tumors and patients who did not undergo RFA or for whom no preinterventional MRI examination was available were not taken into consideration.

\section{Patient group}

In this retrospective case control study, CT-guided RFA to treat an OO was performed during the study period at our institution in $\mathrm{n}=150$ patients. Intraarticular $\mathrm{OO}$ was seen in $\mathrm{n}=24$ patients. An OO was defined as intraarticular if the nidus was within the articular capsule. $\mathrm{n}=3$ patients were excluded since an MRI examination performed prior to RFA was not available. In total there were $\mathrm{n}=21$ patients with intraarticular $\mathrm{OO}$ that fulfilled all inclusion criteria. These patients with intraarticular $\mathrm{OO}$ were initially recorded in a table according to anatomical location by a radiologist with 5 years of musculoskeletal imaging experience. A comparison group of the same size $(n=21)$ with extraarticular $\mathrm{OO}$ in a similar or comparable anatomical location, e. g. femoral location or pelvis or shaft of an extremity bone, was recruited to increase comparability. Patients were selected consecutively with the sole criterion of anatomical comparability while satisfying the other inclusion criteria, particularly the availability of a preinterventional MRI examination. The database was searched starting with the most recent date and the first patient with suitable criteria was selected. Subsequent possibly suitable patients were not taken into consideration. In total, $\mathrm{n}=21$ patients with intraarticular $\mathrm{OO}$ and 21 patients with extraarticular $\mathrm{OO}$ who underwent CT-guided RFA were included in this study.

\section{Clinical presentation}

The local hospital information system (HIS) provided information regarding the clinical presentation at the first visit. This included the patient's pain symptoms prior to RFA. A differentiation was made here between generalized pain and local pain and it was determined whether there was an increase in pain at night or if the pain improved with NSAID intake. Soft-tissue swelling localized around a certain joint was also included in the clinical evaluation prior to RFA. The initial suspected diagnosis was also determined. This included the clinical suspected diagnosis at the time of referral to the orthopedic university hospital based on the clinical examination and the various imaging methods (including ultrasound, X-ray, scintigraphy, and MRI). Radiology reports, documentation of post-treatment (radiological and orthopedic) medical rounds and physician reports regarding outpatient follow-up provided information regarding the clinical course after RFA. This includes the development of pain symptoms and the occurrence of complications.

\section{RFA technique}

CT-guided RFA was performed in all patients with intraarticular and extraarticular $\mathrm{OO}$ using the same standardized technique [16-18]. The intervention was performed under sterile conditions and under general anesthesia. Using multidetector CT including multiplanar reconstructions, the access route was initially planned. After administration of local anesthesia (bupivacaine hydrochloride $0.5 \%$ ) and skin incision, puncture using a coaxial bone biopsy system (Bonopty ${ }^{\circledR}$; AprioMed, Uppsala, Sweden), consisting of a hand drill (length $122 \mathrm{~mm}$, diameter $1.7 \mathrm{~mm}$ ) and a penetration cannula (length $95 \mathrm{~mm}$, internal diameter $1.8 \mathrm{~mm}$, external diameter $2.1 \mathrm{~mm}$ ) was performed. After removal of the placeholder, the ablation electrode was inserted through the hollow needle. To position the active tip within the lesion, thin-slice, multiplanar reconstructions were created. Depending on the size of the lesion, two electrode tips (Cool-tip ${ }^{\mathrm{TM}}$; Valleylab, Tyco Healthcare Group LP, Boulder, CO, USA) of varying length were available $(0.7 \mathrm{~cm}$ and $1.0 \mathrm{~cm})$. Once the optimal needle position was reached, the cannula was partially retracted to prevent heat propagation along the needle. RFA was then started by slowly increasing the power until the target temperature of $90^{\circ} \mathrm{C}$ was reached. The total ablation time for each ablation procedure was always 400 s regardless of the anatomical location. In one patient with intraarticular $\mathrm{OO}$ of the distal femur, thermoprotection of the adjacent retropatellar cartilage was performed. The joint space was punctured with a 22G needle and filled with distilled water to create an insulating layer and to increase the distance with respect to the site of maximum heat generation. In general, patients were discharged within 24 hours after post-treatment medical rounds including a physical examination.

\section{MRI technique and protocol}

MRI examinations were performed using a 70-cm 3 T whole-body open bore MRI scanner (MAGNETOM Verio, Siemens Healthineers, Erlangen, Germany) equipped with an 18-channel total imaging matrix (Tim [102 $\times 18]$ configuration) and various dedicated coils depending on the examined region (e.g. knee coil, flex coil, body coil). All joints were examined using dedicated MRI coils. Each examination followed the same sequence accordingly adapted to the particular examination region. The examination protocol included a T1w sequence without fat saturation, a T2w sequence without fat saturation and a Short-Tau-Inversion-Recovery (STIR) sequence prior to contrast medium (CM) administration and a T1w sequence with and without fat saturation on two planes after CM administration. The T1w sequence without fat saturation was acquired before and after $\mathrm{CM}$ administration on the same plane to allow subtraction imaging. MRI examinations performed at a field strength of 1.5 Tesla and with comparable sequences but different sequence parameters (echo time, repetition time) were available in $\mathrm{n}=10$ patients. Contrast medium was not administered in $\mathrm{n}=8$ patients.

\section{Image analysis}

The image analysis concentrated on a qualitative visual assessment. The presence or absence of effusion/synovitis on MRI was 
evaluated. In the few cases $(n=8)$ in which MRI was performed without contrast, synovitis was evaluated in agreement with other work groups $[19,20]$ based on synovial hypertrophy and concomitant joint effusion. If a contrast-enhanced MRI examination was available, the greater enhancement of the synovialis was used for diagnosis. Moreover, a check for bone marrow edema, soft tissue edema, or a periosteal reaction was performed. A periosteal reaction was defined as T1 and T2 hypointense periosteal thickening or a single layer detached from the cortical bone with or without a T2 hyperintense subperiosteal or paraperiosteal lamella of liquid and corresponding contrast enhancement. In addition, the MRI examinations regarding periosteal reaction were correlated with the preinterventional CT images. Simple continuous or interrupted single-layer periosteal reactions and malignant periosteal reactions, such as spicules, the Codman Triangle, or a sunburst appearance, were differentiated. Detection of the nidus on MRI as well as the contrast enhancement and the signal intensity of the nidus (hypo-, iso-, or hyperintensity compared to the surrounding muscle tissue) were examined as further features. The signal intensity of the nidus on the $\mathrm{T} 2 \mathrm{w}$ sequences was then compared to the degree of sclerosis of the nidus on preinterventional CT. The extent of the sclerosis in relation to the total size of the nidus ( $>50 \%$ and $<50 \%$ ) was evaluated and the density of the nidus (Hounsfield units) was also measured. The evaluation was always performed on the layer with the greatest nidus dimension. If a follow-up examination was available, it was included in the study. All image datasets were available in digital DICOM format on our PACS (image archiving and communication system, GE Centricity EnterpriseTM, Version 4.2.7.4, General Electric Healthcare Pty Ltd. Piscataway, New Jersey, USA) and were evaluated in consensus by two radiologists with 5 and 15 years of musculoskeletal imaging experience.

\section{Statistics}

The statistical evaluation was performed using SAS for Windows version 9.4 (SAS Institute Inc., North Carolina). All analyses are descriptive and explorative. Two-sided p-values were reported and a $5 \%$ significance level was set. A descriptive analysis of the demographic and disease-specific information, pretreatment clinical presentation, morphological characteristics and post-treatment response was performed. The following statistical variables were calculated as continuous features: $n$, mean, standard deviation median, minimum and maximum. Qualitative features were summarized as the absolute and relative frequencies of the individual forms. All features were evaluated for the entire population as well as separately for patients with intraarticular and extraarticular OO. The exact Wilcoxon rank sum test was used to compare age at MRI prior to RFA and the time period of MRI before and after RFA between patients with intraarticular and extraarticular OO. The $\mathrm{Chi}^{2}$ test was used to compare the features of the pretreatment clinical presentation and the pretreatment and posttreatment MRI characteristics between patients with intraarticular $\mathrm{OO}$ and patients with extraarticular $\mathrm{OO}$. If the requirements for the $\mathrm{Chi}^{2}$ test were not met, the Fisher's exact test was used. Frequencies are displayed as grouped bar charts. To evaluate the diagnostic value of effusion/synovitis, bone marrow edema, soft tissue edema, periosteal reaction, contrast enhancement and nidus detection as features to predict intraarticular $\mathrm{OO}$, the sensitivity, specificity, positive and negative predictive value and the exact $95 \%$ confidence intervals were calculated and shown graphically.

\section{Results}

\section{Patient group}

32 male (76.2\%) and 10 female (23.8\%) patients with an average age of 19.4 years (range: $8.0-55.9$ years) were included in the study. An analysis of both the intraarticular $\mathrm{OO}$ group and the extraarticular $\mathrm{OO}$ group with respect to age and gender showed two very homogeneous patient groups. OO of the femur $(n=18$; $42.9 \%)$ and of the humerus $(n=8 ; 19.0 \%)$ was seen most frequently. The demographic data of the patient groups and the $\mathrm{OO}$ location including frequency data are summarized in $>$ Table 1.

\section{Clinical presentation prior to RFA}

An incorrect suspected diagnosis at the time of referral was initially made in $n=14$ patients $(66.7 \%$ ) with intraarticular OO. In contrast, an extraarticular 00 was correctly suspected upon initial presentation in 17 patients $(81.0 \%)$. Thus, the number of false initial diagnoses in the intraarticular 00 group was significantly higher compared to the extraarticular 00 control group $(p=0.0018)$. $>$ Table 2 provides an overview of the different suspected diagnoses and their frequency. All 42 patients complained of local, non-exercise-induced pain prior to treatment. $\mathrm{N}=15 \mathrm{pa}$ tients with intraarticular $\mathrm{OO}(71.4 \%)$ and $\mathrm{n}=18$ patients with extraarticular $\mathrm{OO}(85.7 \%)$ reported pain that increased at night. $\mathrm{N}=2$ patients $(\mathrm{n}=1$ intraarticular $\mathrm{OO}, \mathrm{n}=1$ extraarticular $\mathrm{OO})$ did not experience an increase in pain at night. With respect to pain symptoms prior to RFA, there were no significant differences between intraarticular $\mathrm{OO}$ and extraarticular $\mathrm{OO}$. A response to NSAIDs was reported in $\mathrm{N}=13$ patients with intraarticular $\mathrm{OO}$ $(61.9 \%)$ and $n=14$ patients with extraarticular 00 (66.7\%). There were also no significant differences between the two patient groups in this regard. In total, $\mathrm{n}=5$ patients with intraarticular $\mathrm{OO}$ additionally presented with soft tissue swelling localized around a specific joint (11.9\%), while this finding was not seen in any patients in the extraarticular $\mathrm{OO}$ group $(\mathrm{p}=0.0478)$.

\section{MRI features prior to RFA}

The nidus could be identified on MRI in every case regardless of location. Fig. 1 shows the MRI features prior to RFA and their frequency in the intraarticular and extraarticular 00 groups.

- Fig. 2 shows the resulting quality criteria of MRI characteristics for evaluating intraarticular OO. While effusion and synovitis were observed in all patients with intraarticular $\mathrm{OO}$ ( $>$ Fig. 3c, d, 4a, b), this finding was not seen in anyone in the comparison group. Effusion and synovitis were seen with highly significant greater frequency in the intraarticular 00 group $(p<0.0001)$ and there was perfect sensitivity (100\%) and specificity (100\%) and a higher negative predictive value of $84.6 \%$ with respect to the prediction of intraarticular OO. Both intraarticular and extraarticular OOs showed 
- Table 1 Patients.

\begin{tabular}{|c|c|c|c|c|}
\hline & & intraarticular & extraarticular & total \\
\hline \multirow[t]{2}{*}{ sex } & male & $16(76.2 \%)$ & $16(76.2 \%)$ & 32 \\
\hline & female & $5(23.8 \%)$ & $5(23.8 \%)$ & 10 \\
\hline \multirow[t]{6}{*}{ age (years) } & $\mathrm{n}$ & 21 & 21 & 42 \\
\hline & mean & 25.2 & 20.7 & 23.0 \\
\hline & SD & 14.02 & 8.76 & 11.77 \\
\hline & median & 20.5 & 18.7 & 19.4 \\
\hline & $\min$ & 8.4 & 8.0 & 8.0 \\
\hline & $\max$ & 55.9 & 36.2 & 55.9 \\
\hline \multirow[t]{14}{*}{ localization } & acetabulum & $1(4.8 \%)$ & $1(4.8 \%)$ & 2 \\
\hline & calcaneus & - & 1 (4.8\%) & 1 \\
\hline & femur & $7(33.3 \%)$ & $8(38.1 \%)$ & 15 \\
\hline & glenoid & $1(4.8 \%)$ & - & 1 \\
\hline & humerus & $4(19.0 \%)$ & $4(19.0 \%)$ & 8 \\
\hline & capitate & $1(4.8 \%)$ & - & 1 \\
\hline & cuboid & $1(4.8 \%)$ & - & 1 \\
\hline & hamate & $1(4.8 \%)$ & - & 1 \\
\hline & metacarpale & - & 1 (4.8\%) & 1 \\
\hline & sacrum & $1(4.8 \%)$ & 1 (4.8\%) & 2 \\
\hline & talus & $1(4.8 \%)$ & 1 (4.8\%) & 2 \\
\hline & tibia & $2(9.5 \%)$ & $2(9.5 \%)$ & 4 \\
\hline & ulna & $1(4.8 \%)$ & $1(4.8 \%)$ & 2 \\
\hline & phalanx & - & $1(4.8 \%)$ & 1 \\
\hline
\end{tabular}

concomitant bone marrow edema in all cases (100\%). Bone marrow edema is thus extremely sensitive (100\%) with respect to the detection of intraarticular $\mathrm{OO}$ but has a lower specificity and lower positive and negative predictive values. If contrast medium was administered for the MRI examination, contrast enhancement of the nidus could always be observed ( $\mathbf{F i g .} \mathbf{3 b} \mathbf{b} \mathbf{d}, \mathbf{4 b}$ ). In 8 cases $(19.0 \%)$, contrast was not administered despite a corresponding recommendation, resulting in a high sensitivity (90\%) and a high negative predictive value (75\%) for this feature regarding intraarticular OO. Perifocal soft tissue edema was observed significantly more frequently in the intraarticular 00 group $(n=14$ patients $(66.7 \%)$ ) compared with the extraarticular $\mathrm{OO}$ group ( $\mathrm{n}=6$ patients $(28.6 \%))(p=0.0143)$. However, with a sensitivity of $66.7 \%$ and a specificity of $71.4 \%$, soft tissue edema is not a good quality criterion for evaluating intraarticular $\mathrm{OO}$. A periosteal reaction was seen on MRI in $n=13$ patients $(61.9 \%)$ with intraarticular $\mathrm{OO}$ and $\mathrm{n}=12$ patients $(57.1 \%$ ) with extraarticular $\mathrm{OO}(\triangleright \mathbf{F i g} \mathbf{3 b})$. The periosteal reactions could be confirmed in all cases in a correlation with the available preinterventional CT images ( $\triangleright$ Fig. $4 a, c$ ). In this group only simple continuous single-layer periosteal reactions were seen, while aggressive forms, such as spicules, were not seen. No significant difference between the comparison groups with respect to a periosteal reaction was seen $(p=0.7532)$. The T1w sequences did not show uniform signal intensity of the nidus
- Table 2 Initial diagnosis.

\begin{tabular}{|l|l|l|}
\hline initial diagnosis & intraarticular & extraarticular \\
\hline osteoidosteoma & $7(33.3 \%)$ & $17(81.0 \%)$ \\
\hline indistinct tumor mass & $3(14.3 \%)$ & $2(9.5 \%)$ \\
\hline osteomyelitis & $3(14.3 \%)$ & $1(4.8 \%)$ \\
\hline malignoma & $2(9.5 \%)$ & - \\
\hline posttraumatic pain & $2(9.5 \%)$ & - \\
\hline $\begin{array}{l}\text { epicondylitis radialis } \\
\text { humeri }\end{array}$ & $1(4.8 \%)$ & - \\
\hline meniscal injury & $1(4.8 \%)$ & - \\
\hline CRMO & $1(4.8 \%)$ & - \\
\hline enchondroma & - & $1(4.8 \%)$ \\
\hline chondroblastoma & $1(4.8 \%)$ & - \\
\hline
\end{tabular}

CRMO = Chronic Recurrent Multifocal Osteomyelitis 


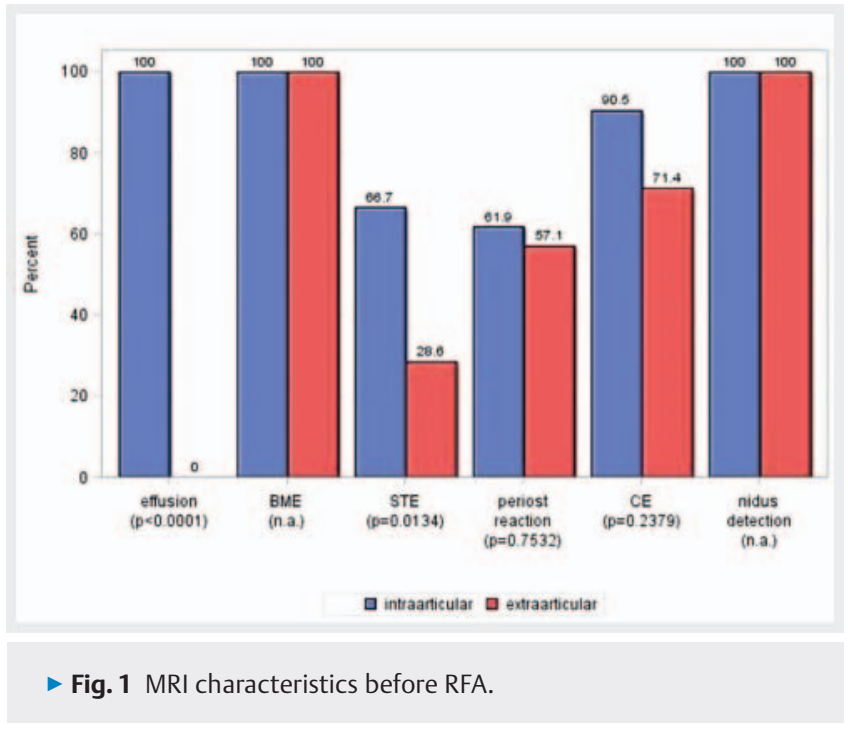

(54.8\% hypointense, $35.7 \%$ isointense, $7.1 \%$ hyperintense). The T2 signal of the nidus was also very heterogeneous ( $16.7 \%$ hypointense, $9.5 \%$ isointense, $73.8 \%$ hyperintense). There was no significant difference regarding signal behavior between intraarticular and extraarticular 00 . The correlation of $\mathrm{T} 2 \mathrm{w}$ signals with the degree of sclerosis of the nidus on CT showed that all cases of intraarticular and extraarticular $\mathrm{OO}$ with sclerosis of the nidus of $<50 \%$ on CT had a hyperintense $(93.4 \%$ ) or isointense $(6.6 \%)$ signal in the T2w sequences. However, in individual OOs with a hyperintense or isointense T2w signal, hypointense segments could be seen in the T2w sequences at sites with focal sclerosis often in the shape of points on CT. In contrast, OOs with sclerosis of the nidus of $>50 \%$ on CT largely showed a hypointense T2w signal (88.9\%). OOs with a hyperintense T2w signal had an average density of $237.4 \mathrm{HU}$ (199-386 HU), while OOs with a hypointense T2w signal had an average density of $528.3 \mathrm{HU}(368-787 \mathrm{HU})$.

\section{MRI characteristics after RFA}

An MRI scan after RFA was available in a subgroup of $n=31$ patients ( $n=17$ patients with intraarticular $00, n=14$ patients with extraarticular 00). The median time between pretreatment and post-treatment MRI examination was 6.4 months (range: 1.525.1 months). In $\mathrm{n}=24$ patients, contrast medium was administered for the follow-up MRI examination. A decrease in bone marrow edema and in the contrast enhancement of the nidus was always observed after RFA in both the intraarticular OO group and the extraarticular $O O$ group. Effusion/synovitis decreased in all patients with intraarticular OO. Effusion and synovitis were not seen in patients with extraarticular 00 even over the course of the disease. If soft tissue edema or a periosteal reaction was present prior to treatment, a decrease after RFA was seen in both the intraarticular $\mathrm{OO}$ group and the extraarticular $\mathrm{OO}$ group. If these findings were not initially present, they were also not seen over the course of the disease. In summary, all MRI findings decreased after treatment with RFA in both groups. The T1 and T2 signal intensities of the nidus remained highly variable even after RFA and did not differ significantly between intraarticular

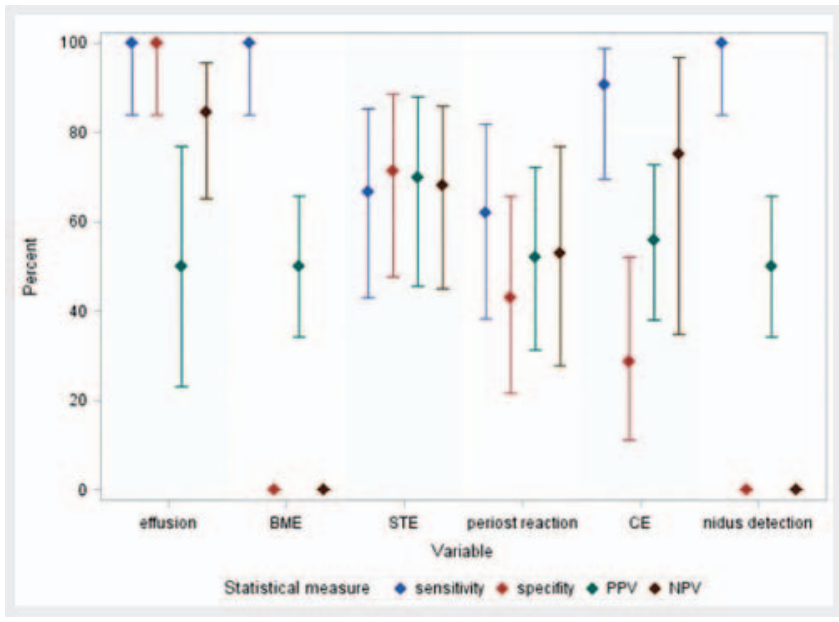

- Fig. 2 Predictive value of MRI characteristics for evaluating Intraarticular Osteoid Osteoma.

and extraarticular OO. Fig. 4 shows an example of a patient from the series with intraarticular 00 of the distal femur with the MRI characteristics before and after RFA.

\section{Clinical course after RFA}

All patients reported a decrease in pain symptoms after RFA. Complications were not observed in any of the cases. The articular cavity of one patient was filled with distilled water to protect the articular cartilage. No post-treatment cartilage damage occurred.

\section{Discussion}

The high rate of false suspected clinical diagnoses in the present study highlights the difficulty of diagnosing intraarticular $\mathrm{OO}$ which usually presents with nonspecific symptoms and atypical radiological signs. The literature also includes numerous case descriptions reporting a high frequency of incorrect diagnoses and a treatment delay of 1 to 10 years $[21,22]$. In this study the nidus could always be identified on high-resolution MRI resulting in the diagnosis of intraarticular $\mathrm{OO}$. This contradicts the results of previous studies in which the nidus could only be detected on CT not MRI in up to $35 \%$ of cases [23]. This shows the technical development of MRI with respect to image quality and resolution so that reliable identification of the nidus is now possible using the corresponding dedicated examination technique. The most important MRI signs for diagnosing an intraarticular $\mathrm{OO}$ are synovitis and joint effusion, which are always present in intraarticular $\mathrm{OO}$ and can be differentiated from extraarticular $\mathrm{OO}$ with perfect sensitivity and specificity and a high negative predictive value. Effusion and synovitis in intraarticular $\mathrm{OO}$ seem to be caused by COX-2 expression of the nidal osteoblasts, resulting in prostaglandin production and thus lymphofollicular inflammation in the perifocal synovial tissue via the arachidonic acid metabolic pathway. [24-26]. Knowledge of this fundamental morphological difference between intraarticular $\mathrm{OO}$ and extraarticular $\mathrm{OO}$ is clinically relevant. Intraarticular $\mathrm{OO}$ should be included in the differential diagnosis particularly in young patients with joint effu- 

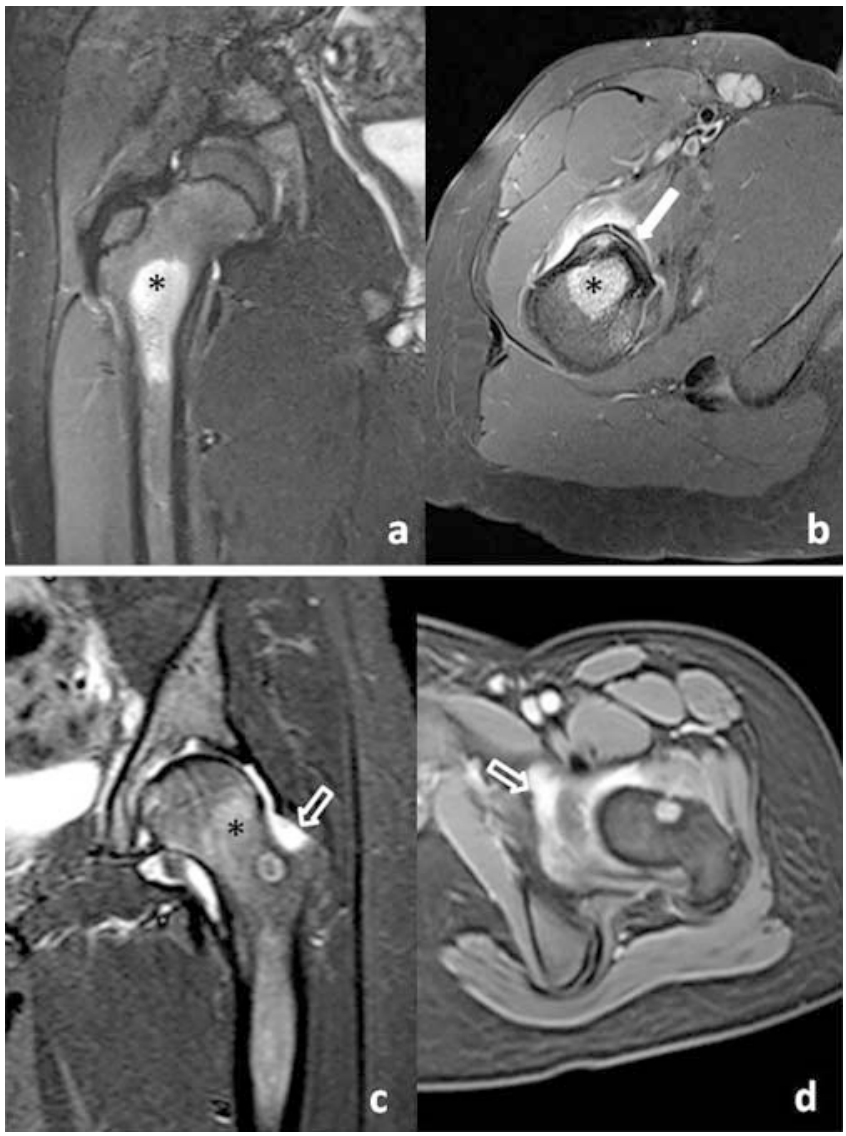

- Fig. 3 a, b 9-year-old patient with extraarticular osteoid osteoma of the right proximal femur; a Significant bone marrow edema can be seen in the coronal STIR (star). There is no joint effusion. $\mathbf{b}$ The nidus shows strong enhancement in the axial $\mathrm{T} 1$ post-CM. In addition, the thickened, $\mathrm{T} 1$ hypointense periosteum, which is lifted from the cortex, is demarcated by a thin, subperiosteal CM uptake in terms of a periosteal reaction (arrow). You can see reactive CM enhancement in the ventral bordering soft tissue. c, d 13-year-old patient with intraarticular osteoid osteoma of the left proximal femur; c The nidus at the femoral neck can be easily identified in the coronal STIR. In addition to perifocal bone edema (star), there is also obvious joint effusion (open arrow). $\mathbf{d}$ In the axial T1 post-CM you can see strong enhancement of the articular capsule like synovitis (open arrow). The nidus shows homogeneous CM uptake.

sion/synovitis. In these cases a targeted search for the nidus should be performed because it is absolutely necessary for the diagnosis of an OO. In this way the common incorrect diagnoses can be avoided. An intraarticular $\mathrm{OO}$ can be practically excluded if signs of synovitis and joint effusion are absent. This means that $\mathrm{OO}$ is not necessarily the diagnosis in the case of intraarticular osteolytic bone lesions without synovitis/joint effusion and other differential diagnoses should be primarily considered. This shows that knowledge of the various MRI characteristics is indispensable for radiologists for differentiating intraarticular $\mathrm{OO}$ from extraarticular $\mathrm{OO}$ and for reliably diagnosing or ruling out intraarticular OO. In the present study and in agreement with previous studies, the nidus was visualized with heterogeneous signal behavior in the T1w and T2w sequences [27]. Differences between intraarticular $\mathrm{OO}$ and extraarticular $\mathrm{OO}$ were not statistically significant
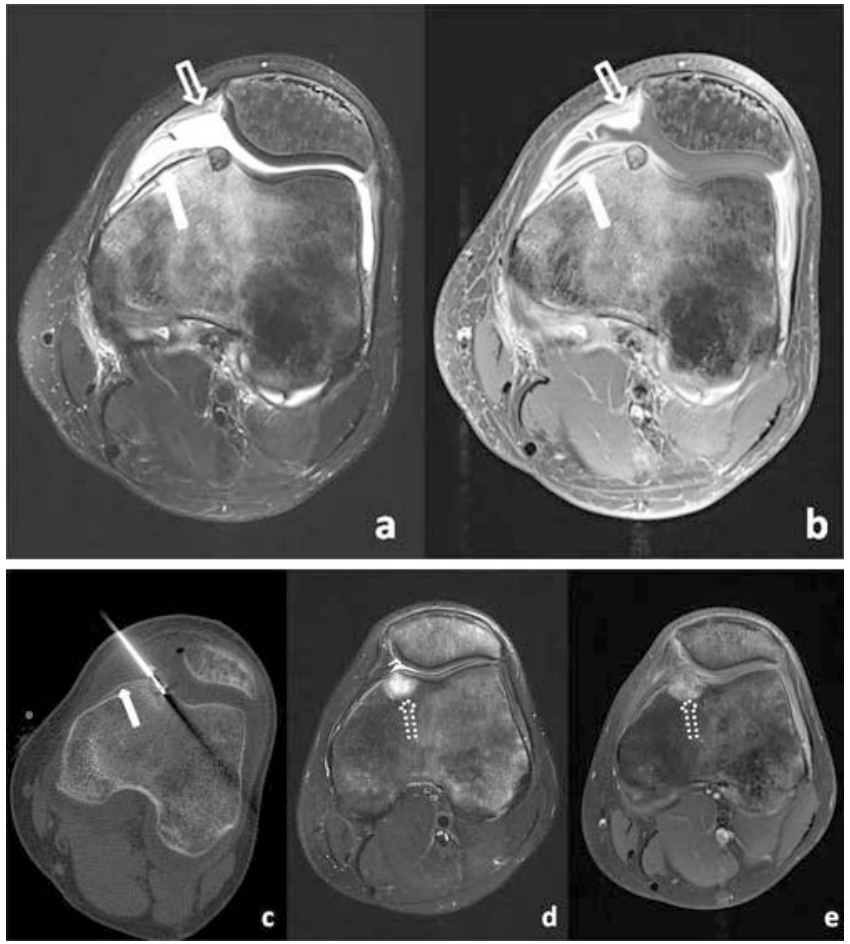

- Fig.4 17-year-old patient with an intraarticular osteoid osteoma of the left distal femur; a, b MRI before RFA. Bone edema and synovial thickening with joint effusion can be seen in the axial T2fs a (open arrow). The axial T1 post-CM $\mathbf{b}$ shows obvious synovial enhancement (open arrow) and CM uptake of the nidus. In both sequences, a hypointense periosteal retraction with subperiosteal fluid accumulation and CM uptake are clearly definable (arrow). c Axial planning CT before RFA. CT-guided RFA was performed with a $7 \mathrm{~mm}$ unipolar RFA electrode and a temperature of $90^{\circ} \mathrm{C}$ over an ablation time of $6 \mathrm{~min}$. To protect the retropatellar cartilage, the articular cavity was inflated with distilled water. The hypersclerosed periosteum (arrow) is demarcated in correlation to the MRI. d, e MRI 3 months after RFA. The nidus can no longer be clearly identified in the axial T2fs $\mathbf{d}$ and axial T1 post-CM e. Joint effusion/synovitis and bone edema are definitely regressive. There is a homogeneously increased T2 signal with correlating CM enhancement in the ablation area (dashed arrow), which is primarily to be seen as post-therapeutic reactive changes.

in relation to signal behavior. In a correlation of the T2w signal with preinterventional planning CT examinations, we were able to determine that OOs with $>50 \%$ sclerosis on CT primarily have hypointense $\mathrm{T} 2 \mathrm{w}$ signals and significantly higher density values compared to OOs with a hyperintense and isointense T2w signal. Thus, the T2w signal seems to be largely dependent on the extent of calcification. This coincides with the results of Allen et al. [2], which state that signal behavior depends on the degree of sclerosis and on age, size and vascularization. On the whole, T1 and T2 signal intensities are heterogeneous and therefore have limited diagnostic value. The nidus exhibited contrast enhancement in all cases in our study in which contrast was administered. Particularly due to the heterogeneous signal behavior in native T1w/T2w sequences, contrast enhancement of the nidus supports the diagnosis, delimits from other differential diagnoses, such as ganglia, and helps to evaluate relapse in the course of the disease [23, 27$29]$. With a sensitivity of $100 \%$ in both groups, bone marrow ede- 
ma is perfectly suited for reliably detecting extraarticular as well as intraarticular $\mathrm{OO}$ on MRI. If bone marrow edema is not present, an OO can be practically ruled out. However, if bone marrow edema is present, a targeted search for further features of an $\mathrm{OO}$ should be performed particularly in young patients. Although perifocal soft tissue edema could be observed more frequently in intraarticular $\mathrm{OO}$, it was not always present. Therefore, it can at best be an indicator of intraarticular $\mathrm{OO}$ but cannot reliably differentiate from extraarticular $\mathrm{OO}$ due to the low specificity. Simple continuous single-layer periosteal reactions occurred in both groups but cannot be used to differentiate between an intraarticular and extraarticular location of an $\mathrm{OO}$ due to the low predictive value. We were able to show that MRI is suitable for identifying an intraarticular $\mathrm{OO}$ and for differentiating from an extraarticular OO. MRI can thus prevent delayed diagnosis and allow early treatment. This is particularly important in growing young patients since an intraarticular $\mathrm{OO}$ can lead to chronic damage to the articular cartilage and bony deformities [30]. CT-guided RFA is a safe and effective treatment for $\mathrm{OO}$, resulting in a decrease in pathological MRI findings and excellent clinical results [16-18]. Moreover, promising results regarding high-frequency ultrasound treatment have also been achieved [31]. In the case of an intraarticular $\mathrm{OO}$ in a critical location with respect to the articular cartilage, protective measures, e.g. creation of artificial joint effusion, could reduce the risk of post-treatment articular defects. A clear decrease in both pain symptoms and the morphological changes visible on MRI after RFA was also seen in the present study.

\section{Limitations}

The present study has a number of limitations to be taken into consideration when formulating possible conclusions. Since this is a retrospective study for which two equal-sized patient groups with similar $\mathrm{OO}$ locations were formed to ensure the comparability of MRI characteristics of intraarticular and extraarticular OOs, there could be a selection bias. Randomization could not be performed due to the retrospective nature of the study. A further limitation is the lack of a healthy control group. However, the main goal of this study was to compare intraarticular and extraarticular OOs with respect to their clinical presentation and MRI features. The MRI examinations were performed at different times in the course of the disease so that it is not possible to make any statements as to when certain features occur. A standard examination protocol was not used in all cases and MRI examinations were performed without the administration of contrast medium in $n=8$ patients despite a corresponding recommendation. A standard examination protocol would improve the validity of our results. When evaluating synovitis on MRI without contrast, the finding was "no synovitis" if effusion was absent and no synovial thickening could be detected. In these cases, it is theoretically possible that synovitis can only be visualized by contrast enhancement resulting in a false-negative finding. A further limitation of this study is the lack of histological confirmation of OO. However, in consensus with many other work groups, this is not absolutely necessary for diagnosis when the clinical and morphological presentation is typical $[15,16]$. The patient group ( $n=42$ patients) is relatively small but the intraarticular 00 group is the largest described to date.

\section{CLINICAL RELEVANCE OF THE STUDY}

- The clinical diagnosis of intraarticular OO is often problematic and other differential diagnoses are primarily considered. As a result, correct diagnosis is often delayed, clinical pain symptoms are prolonged and proper treatment is delayed.

- MRI is highly suited for diagnosing extraarticular as well as intraarticular OO. Joint effusion and synovitis are always present in intraarticular $\mathrm{OO}$ and differentiate between the two types with perfect sensitivity/specificity.

- Particularly in young/adolescent patients with joint effusion and typical pain symptoms, intraarticular $O O$ should therefore always be considered. It can be diagnosed based on visualization of the nidus and perifocal bone and soft tissue edema on MRI.

- All MRI changes indicating activity, like synovitis, contrast enhancement of the nidus, bone marrow and soft tissue edema, decrease after successful RFA.

- The clinical results after RFA are excellent in both forms.

\section{Conflict of Interest}

The authors declare that they have no conflict of interest.

\section{References}

[1] Lee EH, Shafi M, Hui JH. Osteoid osteoma: a current review. J Pediatr Orthop 2006; 26: 695-700. doi:10.1097/01.bpo.0000233807.80046.7c

[2] Allen SD, Saifuddin A. Imaging of intra-articular osteoid osteoma. Clin Radiol 2003; 58: 845-852

[3] May C], Bixby SD, Anderson ME et al. Osteoid Osteoma About the Hip in Children and Adolescents. J Bone Joint Surg Am 2019; 101: 486-493. doi:10.2106/JBJS.18.00888

[4] Norman A, Abdelwahab IF, Buyon J et al. Osteoid osteoma of the hip stimulating an early onset of osteoarthritis. Radiology 1986; 158: 417420. doi:10.1148/radiology.158.2.3941866

[5] Weber KL, Morrey BF. Osteoid osteoma of the elbow: a diagnostic challenge. J Bone Joint Surg Am 1999; 81: 1111-1119

[6] Snow SW, Sobel M, DiCarlo EF et al. Chronic ankle pain caused by osteoid osteoma of the neck of the talus. Foot Ankle Int 1997; 18: 98-101. doi:10.1177/107110079701800211

[7] Sprengel SD, Weber MA, Lehner B et al. Osteoidosteoma. From diagnosis to treatment. Radiologe 2015; 55: 479-486. doi:10.1007/s00117-0142805-5

[8] Szendroi M, Kollo K, Antal I et al. Intraarticular osteoid osteoma: clinical features, imaging results, and comparison with extraarticular localization. J Rheumatol 2004; 31: 957-964

[9] Frassica F], Waltrip RL, Sponseller PD et al. Clinicopathologic features and treatment of osteoid osteoma and osteoblastoma in children and adolescents. Orthop Clin North Am 1996; 27: 559-574

[10] Firooznia H, Rafii M, Golimbu C. Computed tomography of osteoid osteoma. J Comput Tomogr 1985; 9: 265-268

[11] Pikoulas C, Mantzikopoulos G, Thanos L et al. Unusually located osteoid osteomas. Eur J Radiol 1995; 20: 120-125 
[12] Mommert I, Heuschmidt M, Suckel A. Intraarticular osteoid osteoma as a cause of chronic ankle pain. Orthopade 2009; 38: 269-273. doi:10.1007/s00132-009-1412-z

[13] Filippiadis DK, Velonakis G, Kostantos C et al. Computed tomographyguided radiofrequency ablation of intra-articular osteoid osteoma: a single centre's experience. Int J Hyperthermia 2017; 33: 670-674. doi:10.1080/02656736.2017.1294711

[14] Heuck A, Reiser M, Lehner K. Imaging of osteoid osteoma by MR tomography. Radiologe 1988; 28: 522-527

[15] Vanderschueren GM, Taminiau AH, Obermann WR et al. Osteoid osteoma: clinical results with thermocoagulation. Radiology 2002; 224: 8286. doi:10.1148/radiol.2241011135

[16] Rehnitz C, Sprengel SD, Lehner B et al. CT-guided radiofrequency ablation of osteoid osteoma and osteoblastoma: clinical success and longterm follow up in 77 patients. Eur J Radiol 2012; 81: 3426-3434. doi:10.1016/j.ejrad.2012.04.037

[17] Weber MA, Sprengel SD, Omlor GW et al. Clinical long-term outcome, technical success, and cost analysis of radiofrequency ablation for the treatment of osteoblastomas and spinal osteoid osteomas in comparison to open surgical resection. Skeletal Radiol 2015; 44: 981-993. doi:10.1007/s00256-015-2139-z

[18] Rehnitz C, Sprengel SD, Lehner B et al. CT-guided radiofrequency ablation of osteoid osteoma: correlation of clinical outcome and imaging features. Diagn Interv Radiol 2013; 19: 330-339. doi:10.5152/ dir.2013.096

[19] Hill CL, Hunter DJ, Niu J et al. Synovitis detected on magnetic resonance imaging and its relation to pain and cartilage loss in knee osteoarthritis. Ann Rheum Dis 2007; 66: 1599-1603. doi:10.1136/ard.2006.067470

[20] Loeuille D, Sauliere N, Champigneulle ] et al. Comparing non-enhanced and enhanced sequences in the assessment of effusion and synovitis in knee OA: associations with clinical, macroscopic and microscopic features. Osteoarthritis Cartilage 2011; 19: 1433-1439. doi:10.1016/ j.joca.2011.08.010

[21] Franceschi F, Marinozzi A, Papalia R et al. Intra- and juxta-articular osteoid osteoma: a diagnostic challenge: misdiagnosis and successful treatment: a report of four cases. Arch Orthop Trauma Surg 2006; 126 : 660-667. doi:10.1007/s00402-006-0203-9
[22] Francesco B, Andrea LA, Vincenzo S. Intra-articular osteoid osteoma of the lower extremity: diagnostic problems. Foot Ankle Int 2002; 23: $264-$ 267. doi:10.1177/107110070202300314

[23] Davies M, Cassar-Pullicino VN, Davies AM et al. The diagnostic accuracy of MR imaging in osteoid osteoma. Skeletal Radiol 2002; 31: 559-569. doi:10.1007/s00256-002-0546-4

[24] Mungo DV, Zhang X, O'Keefe RJ et al. COX-1 and COX-2 expression in osteoid osteomas. J Orthop Res 2002; 20: 159-162. doi:10.1016/ S0736-0266(01)00065-1

[25] Kawaguchi Y, Sato C, Hasegawa T et al. Intraarticular osteoid osteoma associated with synovitis: a possible role of cyclooxygenase-2 expression by osteoblasts in the nidus. Mod Pathol 2000; 13: 1086-1091. doi:10.1038/modpathol.3880202

[26] Snarr JW, Abell MR, Martel W. Lymphofollicular synovitis with osteoid osteoma. Radiology 1973; 106: 557-560. doi:10.1148/106.3.557

[27] Assoun J, Richardi G, Railhac J] et al. Osteoid osteoma: MR imaging versus CT. Radiology 1994; 191: 217-223. doi:10.1148/ radiology.191.1.8134575

[28] Teixeira PA, Chanson A, Beaumont M et al. Dynamic MR imaging of osteoid osteomas: correlation of semiquantitative and quantitative perfusion parameters with patient symptoms and treatment outcome. Eur Radiol 2013; 23: 2602-2611. doi:10.1007/s00330-013-2867-1

[29] von Kalle T, Langendorfer M, Fernandez FF et al. Combined dynamic contrast-enhancement and serial 3D-subtraction analysis in magnetic resonance imaging of osteoid osteomas. Eur Radiol 2009; 19: 25082517. doi:10.1007/s00330-009-1430-6

[30] Song MH, Yoo W], Cho T] et al. Clinical and radiological features and skeletal sequelae in childhood intra-/juxta-articular versus extra-articular osteoid osteoma. BMC Musculoskelet Disord 2015; 16: 3. doi:10.1186/s12891-015-0456-y

[31] Arrigoni F, Napoli A, Bazzocchi A et al. Magnetic-resonance-guided focused ultrasound treatment of non-spinal osteoid osteoma in children: multicentre experience. Pediatr Radiol 2019; 49: 1209-1216. doi:10.1007/s00247-019-04426-0 